\title{
EXISTENCE THEOREMS FOR SOLUTIONS OF DIFFERENTIAL EQUATIONS OF NON-INTEGRAL ORDER*
}

\author{
EVERETT PITCHER AND W. E. SEWELL
}

1. Introduction. In this paper we prove theorems on the existence and uniqueness of solutions of the differential equation

$$
D_{x}^{\alpha} y=\phi(x, y), \quad \alpha>0,
$$

where $\phi(x, y)$ is a known function, $y(x)$ is an unknown function, and $D_{x}^{\alpha} y$ is the Riemann-Liouville $\dagger$ generalized derivative of order $\alpha$ of the function $y(x)$. For $\alpha=1$ the equation (1.1) is an ordinary differential equation of the first order and the restrictions on $\phi(x, y)$ for non-integral $\alpha$ are found to be quite similar to those imposed on the function in the integral case.

In establishing the fundamental existence theorem we first prove (\$2) a theorem of the kind considered by Birkhoff and Kellogg. Our proof rests on three lemmas which are contained in $\$ 3$ along with the definition of the generalized derivative. In $\$ 4$ we establish the existence of a unique solution in the small for $0<\alpha<1$. The extension of this solution throughout the region of definition of $\phi(x, y)$ and the case $\alpha>1$ are considered in $\$ \S 5$ and 6 respectively.

2. The general existence theorem. For our purposes the following theorem is fundamental:

THEOREM 2.1. Let $E$ be a set of continuous functions defined on a common closed interval, and such that if a sequence of functions each belonging to $E$ is uniformly convergent, then the limiting function belongs to $E$ also. Let $S$ be an operator such that if $y$ is in $E$, then $S y$ is in $E$,

* Presented to the Society, September 10, 1937.

$\dagger$ B. Riemann, Gesammelte Mathematische Werke und Wissenschaftlicher Nachlass, Leipzig, 1892, pp. 331-344; J. Liouville, Sur quelques questions de géométrie et de mécanique, et un nouveau genre de calcul pour résoudre ces questions, Journal de l'École Polytechnique, (1), vol. 13, no. 21 (1832), pp. 1-69. For further references see W. E. Sewell, Generalized derivatives and approximation by polynomials, Transactions of this Society, vol. 41 (1937), pp. 84-123; we refer to this paper as SI. See also W. Fabian, Expansions by the fractional calculus, Quarterly Journal of Mathematics, Oxford Series, vol. 7 (1936), pp. 252-255, where other references are given.

$\ddagger$ G. D. Birkhoff and O. D. Kellogg, Invariant points in function space, Transactions of this Society, vol. 23 (1922), pp. 96-115. 
and such that there are constants $\alpha$ and $B$ between 0 and 1 for which, with $y_{1}$ and $y_{2}$ in $E$, we have

$$
\left|S y_{1}-S y_{2}\right| \leqq B \max \left|y_{1}-y_{2}\right|^{\alpha} \text {. }
$$

\section{Then the equation}

$$
y=S y
$$

has a unique solution in $E$.

Let $y_{0}$ denote a function in $E$. Define $y_{n}$ inductively by the equation

$$
y_{n}=S y_{n-1}, \quad n=1,2, \cdots,
$$

and consider the series

$$
y_{0}+\left(y_{1}-y_{0}\right)+\left(y_{2}-y_{1}\right)+\cdots .
$$

From (2.1) and (2.3) we have

$$
\left|y_{n}-y_{n-1}\right| \leqq B \max \left|y_{n-1}-y_{n-2}\right|^{\alpha},
$$

and this recurrence yields

$$
\left|y_{n}-y_{n-1}\right| \leqq B^{1+(n-2) \alpha} K^{m}
$$

where $K=\max \left|y_{1}-y_{0}\right|$ and $m=\alpha^{n-1}$. The test ratio of the series whose general term is the right member of (2.6) is $B^{\alpha} K^{\theta}$ where $\theta=\alpha^{n}-\alpha^{n-1}=\alpha^{n-1}(\alpha-1)$. The factor $B^{\alpha}$ is less than 1 and $\theta$ approaches 0 as $n$ becomes infinite; consequently series (2.4) converges uniformly.

The function $y$ to which (2.4) converges is a solution of (2.2). For

$$
\left|S y_{n}-S y\right| \leqq B \max \left|y-y_{n}\right|^{\alpha}
$$

and hence $S y_{n}$ approaches $S y$ as $n$ becomes infinite and passage to the limit in (2.3) yields (2.2).

If $z$ is a second solution of (2.2) in $E$, then

$$
|y-z|=|S y-S z| \leqq B \max |y-z|^{\alpha} \text {. }
$$

Using (2.7) as a recurrence, we have $y \equiv z$.

3. Definitions and lemmas. Let $f(x)$ be a real-valued function defined on an interval ${ }^{*}[k, l]$. The derivative of $f(x)$ of order $\alpha$, denoted by $D_{x}^{\alpha} f(x)$, is defined by the following equations: $\dagger$

$$
\left(3.1^{\prime}\right) \quad D_{x}^{0} f(x)=f(x) \text {; }
$$

* We use the square brackets to denote a closed interval.

$\dagger[\alpha]$ is the largest integer $\leqq \alpha$. 


$$
\begin{array}{llr}
\left(3.1^{\prime \prime}\right) & D_{x}^{\alpha} f(x)=\frac{1}{\Gamma(-\alpha)} \int_{k}^{x}(x-z)^{-\alpha-1} f(z) d z, & \alpha<0 ; \\
\left(3.1^{\prime \prime \prime}\right) & D_{x}^{\alpha} f(x)=\frac{d^{p}}{d x^{p}} D_{x}^{\alpha-p} f(x), & 0 \leqq[\alpha]=p-1 .
\end{array}
$$

It is to be understood in $\left(3.1^{\prime \prime}\right)$ and $\left(3.1^{\prime \prime \prime}\right)$ that $D_{x}^{\alpha} f(x)$ is defined if and only if the various operations in the order indicated are convergent in the usual sense. In $\left(3.1^{\prime \prime}\right)$ the value of the derivative at $x=k$ is 0 . When $\alpha$ is a positive integer, $D_{x}^{\alpha} f(x)$ in $\left(3.1^{\prime \prime \prime}\right)$ is the ordinary $\alpha$ th derivative of $f(x)$. If $\alpha$ is not an integer, we take that branch of $(x-z)^{-\alpha-1}$ which is real and positive for $x-k$ positive.

If $f(x)$ is bounded and Riemann integrable on $[k, k+h]$, then for $\alpha$ positive $D_{x}^{-\alpha} f(x)$ exists and is continuous. The value of the derivative at $x=k$ is 0 .

Our method of proof depends upon the fact that $D_{x^{\alpha}}$ and $D_{x}^{-\alpha}$ are inverse operations under suitable restrictions. Thus we need the following three lemmas. In all three we restrict $\alpha$ to lie between 0 and 1 .

Lemma 3.1. If $f(x)$ is continuous and possesses a continuous derivative $D_{x}^{\alpha} f(x)$ on an interval $[k, k+h]$, then $f(k)=0$.

Lemma 3.2. If $f(x)$ is continuous and possesses a bounded derivative $g(x)=D_{x}^{\alpha} f(x)$ on an interval $[k, k+h]$ then $D_{x}^{-\alpha} g(x)$ exists on $[k, k+h]$ and is equal to $f(x)$.

Lemma 3.3. If $f(x)$ is continuous on an interval $[k, k+h]$, then $D_{x}^{\alpha} D_{x}^{-\alpha} f(x)$ exists on $[k, k+h]$ and is equal to $f(x)$.

We prove Lemma 3.1 as follows. By definition

$$
D_{x}^{\alpha} f(x)=\frac{d}{d x} D_{x}^{\alpha-1} f(x) .
$$

Writing the indicated derivative for $x=k$ as the limit of a difference quotient, we have

$$
\lim _{x=k} \frac{1}{(x-k) \Gamma(1-\alpha)} \int_{k}^{x}(x-z)^{-\alpha} f(z) d z
$$

which becomes, under the transformation $u=(z-k) /(x-k)$,

$$
\lim _{x=k} \frac{(x-k)^{-\alpha}}{\Gamma(1-\alpha)} \int_{0}^{1}(1-u)^{-\alpha} f[k+u(x-k)] d u .
$$

Applying the First Mean Value Theorem for the integral of a product and carrying out the resulting indicated integration, we have 


$$
\lim _{x=k} \frac{(x-k)^{-\alpha}}{\Gamma(1-\alpha)} \frac{f(\xi)}{1-\alpha},
$$$$
k<\xi<x .
$$

Since $(x-k)^{-\alpha}$ becomes infinite, it follows that $f(\xi)$ approaches 0 , and thus Lemma 3.1 is proved.

Lemma 3.2 follows from Theorem 13.2 of SI. It should be noted here that the hypothesis $f(k)=0$ in Theorem 13.2 is satisfied by virtue of Lemma 3.1.

Lemma 3.3 is included in Theorem 13.1 of SI.

As stated in SI the hypotheses and conclusions of Theorems 13.1 and 13.2 concern subsets of $[k, k+h]$ but it is easy to see that the stronger conclusions follow from our broader hypotheses.

4. The existence theorem in the small for the case $0<\alpha<1$. Let the function $\phi(x, y)$ be defined and continuous in a region $R$ of the $(x, y)$-plane. Let $p(x)$ be defined and continuous at any point $x$ which is the projection of a point of $R$, and let the point $(k, p(k))$ lie in $R$.

In the following lemma $M, A, a, b$, and $h$ are positive constants and $0<\alpha<1$. Furthermore we choose $a$ and $b$ such that points $(x, y)$ for which $0 \leqq x-k \leqq a$ and $|y-p(k)| \leqq b$ belong to $R$, and we take $h$ less than all of the three numbers

$$
a, \quad\left[\frac{\Gamma(1+\alpha) b}{A}\right]^{1 / \alpha}, \quad\left[\frac{\Gamma(1+\alpha)}{M}\right]^{1 / \alpha} .
$$

We note for future use that

$$
\frac{A h^{\alpha}}{\Gamma(1+\alpha)} \leqq b
$$

and that the number $B$ defined by the equation

$$
\frac{M h^{\alpha}}{\Gamma(1+\alpha)}=B
$$

is between 0 and 1.

We are now in position to state this lemma:

Leмма 4.1. If, for all points $(x, y)$ and $\left(x, y^{\prime}\right)$ of $R$,

$$
\begin{aligned}
|\phi(x, y)| & \leqq A \\
\left|\phi(x, y)-\phi\left(x, y^{\prime}\right)\right| & \leqq M\left|y-y^{\prime}\right|^{\alpha},
\end{aligned}
$$

then the equation

$$
y=p(x)+D_{x}^{-\alpha} \phi(x, y)
$$


has a unique continuous solution $y(x)$ on the interval $[k, k+h]$. This solution has the property that $y(k)=p(k)$.

We shall prove the lemma by applying Theorem 1.1. To that end we let $E$ denote the set of all continuous functions $y(x)$ defined on the interval $[k, k+h]$ and satisfying the inequality

$$
|y(x)-p(x)| \leqq b .
$$

We define the operation $S$ on functions $y$ in $E$ by the relation

$$
S y \equiv p(x)+D_{x}^{-\alpha} \phi(x, y) \text {. }
$$

The function $S y$ is in $E$. For $S y$ is a continuous function of $x$ and

$$
\begin{aligned}
|S y-p(x)| & =\left|D_{x}^{-\alpha} \phi(x, y)\right| \\
& \leqq \frac{1}{\Gamma(\alpha)} \int_{k}^{x}(x-z)^{\alpha-1}|\phi[z, y(z)]| d z \\
& \leqq \frac{A}{\Gamma(\alpha)} \frac{(x-k)^{\alpha}}{\alpha} \\
& \leqq b
\end{aligned}
$$

In the above appraisal we obtain (4.10) from (4.9) by using (4.3) and carrying out the integration. We then replace $x-k$ by its upper bound $h$ and use inequality (4.1).

For any two functions $y_{1}$ and $y_{2}$ in $E$ we have

(4.12) $\left|S y_{1}-S y_{2}\right| \leqq B \max \left|y_{1}-y_{2}\right|^{\alpha}$.

For

$$
\begin{aligned}
\left|S y_{1}-S y_{2}\right| & \leqq \frac{1}{\Gamma(\alpha)} \int_{k}^{x}|x-z|^{\alpha-1}\left|\phi\left[z, y_{1}(z)\right]-\phi\left[z, y_{2}(z)\right]\right| d z \\
& \leqq \frac{M \max \left|y_{1}-y_{2}\right|^{\alpha}}{\Gamma(\alpha)} \frac{(x-k)^{\alpha}}{\alpha} \\
& \leqq B \max \left|y_{1}-y_{2}\right|^{\alpha} .
\end{aligned}
$$

We obtain (4.14) by using (4.4) and carrying out the integration. Then we replace $x-k$ by its upper bound $h$ and substitute from (4.2).

The hypotheses of Theorem 2.1 are satisfied and the conclusion is that equation (4.5) has a unique solution $y(x)$ in $E$. It is obvious that $y(k)=p(k)$. We observe that any continuous solution of (4.5) on $[k, k+h]$ is in $E$. For if $y$ is a solution its value is given by $S y$ in (4.7) and the argument that $S y$ is in $E$ still holds. 
Using the notation of the lemma and setting $p(x) \equiv 0$ we have the following theorem:

TheOREM 4.2. If $\phi(x, y)$ satisfies (4.3) and (4.4) and $0<\alpha<1$, then the equation

$$
D_{x}^{\alpha} y=\phi(x, y)
$$

has a unique solution $y=u(x)$ on the interval $[k, k+h]$. Furthermore $u(k)=0$.

From Lemmas 3.2 and 3.3 it is clear that the set of solutions of equation (4.16) and the set of solutions of the equation

$$
y=D_{x}^{-\alpha} \phi(x, y)
$$

on the interval $[k, k+h]$ are identical. Thus we have Theorem 4.2 by applying Lemma 4.1 .

5. The existence theorem in the large. The solution $u(x)$ of equation (4.16) can be extended to the boundary of the region $R$. This is stated precisely in the following theorem:

THEOREM 5.1. There is a number $\zeta($ possibly $+\infty)$ with the following properties:

(1) The solution $u(x)$ of equation (4.16) whose existence is affirmed in Theorem 4.2 can be extended so as to be defined on the interval $k \leqq x<\zeta$.

(2) No limit as $x$ approaches $\zeta$ of points $(x, u(x))$ belongs to $R$.

The method of proof of this theorem is similar to a method sometimes used in proving the corresponding extension to the existence theorem for ordinary differential equations. In brief, let $S_{\epsilon}$ be the region consisting of points of $R$ to the left of the line $x=1 / \epsilon$ whose distance from points of the boundary of $R$ exceeds $\epsilon$. We suppose that $\epsilon$ is positive and so small that $(k, 0)$ belongs to $S_{\epsilon}$. We observe that for any point $(x, y)$ of $S_{\epsilon}$ the numbers $a, b$ of $\$ 4$ may both be taken as $\epsilon / 2$ and consequently a choice $h^{*}$ of $h$ can be made uniformly for points of $S_{\epsilon}$.

We need the following lemma.

LEMMA 5.2. If $u(x)$ is the unique solution of

$$
D_{x}^{\alpha} y=\phi(x, y)
$$

on an interval $[k, l]$ with all points $(x, u(x))$ lying in $S_{\epsilon}$, then $u(x)$ can be extended uniquely as a solution of (5.1) on the interval $\left[k, l+h^{*}\right]$. 
The equation

$$
y=D_{x}^{-\alpha} \phi(x, y)
$$

is an equivalent form of (5.1). We observe that solving (5.2) on $\left[k, l+h^{*}\right]$ is equivalent to solving the following pair of equations:

$$
\begin{array}{rlr}
y= & \frac{1}{\Gamma(\alpha)} \int_{k}^{x}(x-z)^{\alpha-1} \phi[z, y(z)] d z, & k \leqq x \leqq l, \\
y= & \frac{1}{\Gamma(\alpha)} \int_{k}^{l}(x-z)^{\alpha-1} \phi[z, y(z)] d z \\
& +\frac{1}{\Gamma(\alpha)} \int_{l}^{x}(x-z)^{\alpha-1} \phi[z, y(z)] d z, & l \leqq x \leqq l+h^{*}
\end{array}
$$

Equation $\left(5.3^{\prime}\right)$ has the unique solution $u(x)$. We write $u(z)$ for $y(z)$ in the first integral of $\left(5.3^{\prime \prime}\right)$, denoting the resulting function by $p(x)$. Then $\left(5.3^{\prime \prime}\right)$ reduces to

$$
y=p(x)+{ }_{l} D_{x}^{-\alpha} \phi(x, y), \quad l \leqq x \leqq l+h^{*},
$$

where the subscript $l$ indicates that the lower limit of integration in the derivative is $l$. Equation (5.4) has a unique solution by virtue of Lemma 4.1 and the proof is complete.

By Lemma 5.2 the solution of (5.1) whose existence is established in Theorem 3.1 can be extended to the boundary of $S_{\epsilon}$. Since $\epsilon$ can be chosen arbitrarily small, Theorem 5.1 follows.

6. The differential equation of order greater than 1. The equation

$$
D_{x}^{\beta} y=\phi(x, y) \text {, }
$$

where $\beta>1$, can be handled similarly. We consider this case very briefly. Suppose $p=1+[\beta]$. We set $\alpha=\beta-p+1$. Then equation (6.1) can be written

$$
\frac{d^{p-1}}{d x^{p-1}} D_{x}^{\alpha} y=\phi(x, y) .
$$

Any solution $y(x)$ of (6.2) is a solution of

$$
\begin{aligned}
D_{x}^{\alpha} y=a_{0} & +\frac{a_{1}}{1 !} x+\cdots+\frac{a_{p-2}}{(p-2) !} x^{p-2} \\
& +\int_{k}^{x} d z_{1} \int_{k}^{z_{1}} d z_{2} \cdots \int_{k}^{z_{p-2}} d z_{p-1} \phi\left[z_{p-1}, y\left(z_{p-1}\right)\right],
\end{aligned}
$$


for constants $a_{i}$ properly chosen. Conversely, for any choice of constants $a_{i}$ any solution of (6.3) is a solution of (6.2). If $\left(k, a_{0}\right)$ is in $R$ and (4.3) and (4.4) are satisfied, it can be shown by applying Theorem 2.1 that equation (6.3) has a unique solution on some interval $[k, k+h]$ and that

$$
y^{\alpha+i}(k)=a_{i}, \quad i=0, \cdots, p-2,
$$

where $y^{\alpha+i}$ is the derivative of order $\alpha+i$. This leads to the following theorem:

Theorem 6.1. If $\beta, \alpha, p$ are numbers as described above, if $\phi(x, y)$ satisfies (4.3) and (4.4), and if $a_{0}, a_{1}, \cdots, a_{p-2}$ is any set of numbers with $\left(k, a_{0}\right)$ in $R$, then the equation

$$
D_{x}^{\beta} y=\phi(x, y)
$$

has a unique solution satisfying the initial conditions (6.4).

HARVARD UNIVERSITY AND

Georgia School of Technology

\section{NOTE ON INTEGRABILITY CONDITIONS OF IMPLICIT DIFFERENTIAL EQUATIONS*}

\section{CLYDE M. CRAMLET}

The Riquier $\dagger$ theory for computing the integrability conditions of a system of partial differential equations of arbitrary order but in a special form gives a precise method for calculating these conditions without repetitions and for obtaining the initial determinations of the solutions. These general arguments imply a corresponding theorem for implicit systems of equations. It is the purpose of the present note to state that theorem and to point out that it is a consequence of the general theory. All references will be to the Janet exposition.

Let $F^{k},(k=1,2, \cdots, m)$, represent a system of differential equa-

* Presented to the Society, December 28, 1934.

†C. Riquier, Les Systèmes d'Équations aux Dérivées Partielles, Paris, 1910. M. Janet, Les systèmes d'équations aux dérivées partielles, Journal de Mathématiques, (8), vol. 3 (1920), pp. 65-151. J. M. Thomas, Riquier's existence theorems, Annals of Mathematics, vol. 30 (1929), pp. 285-310. J. F. Ritt, American Mathematical Society Colloquium Publications, vol. 14, chap. 9. 